\title{
Response Function of Thallium-Activated Sodium-Iodide Scintillation Counters*
}

\author{
Martin J. Berger and J. Doggett
}

\begin{abstract}
Measurements of gamma rays with $\mathrm{NaI}(\mathrm{Tl})$ crystals yield pulse height distributions related to the true energy spectrum by an integral equation whose kernel (response function) is the probability that an incident photon of energy $E$ will give rise to a pulse of size $E^{\prime}$. The response function has been calculated by the Monte Carlo method for photons with energies from 0.279 to $4.45 \mathrm{Mev}$. Results are presented for cylindrical crystals ranging in size from 0.25 (radius) by 0.5 inch (length) to 2.5 by 9 inches. They are based on the analysis of 50,000 photon histories sampled with the use of the Standards Electronic Automatic Computer (SEAC). Analytical corrections were made for the escape of annihilation radiation and bremsstrahlung from the crystal.
\end{abstract}

\section{Introduction}

The response function of a $\mathrm{NaI}(\mathrm{Tl})$ crystal spectrometer relates the observed pulse height distribution to the true gamma-ray spectrum. The primary means of determining this function is, and presumably will remain, experimental. But a good theoretical understanding will contribute toward improving scintillation spectrometry.

While the theory of the diffusion of gamma radiation in an infinite medium is now well developed, the calculation of the response function is a boundary problem whose exact solution is beyond the scope of theory in its present state.

The subject of this article is a Monte Carlo calculation of the response function, i. e., an experiment carried out on paper. While this falls short of an analytical theory, it is relatively simple to do, and has important advantages compared to a physical experiment. One is not hampered by the limited availability of monoenergetic sources nor by accidental disturbing effects such as background radiation.

Exploratory Monte Carlo calculations of the response function have already been made by Campbell and Boyle ${ }^{1}$ for energies $E \geqslant 6 \mathrm{Mev}$, and by Foote and Koch ${ }^{2}$ for $E \geqslant 4.45$ Mev. An approximate analytical calculation for $E \leqslant 1.3 \mathrm{Mev}$ was carried out by Maeder, Müller, and Wintersteiger. ${ }^{3}$ These authors computed the effect of the first interaction of the incident photon in the crystal exactly and made an elaborate estimate of the effect of multiple interactions. This approximation limits the validity of their considerations to small crystals not much larger than one mean free path of the incident radiation. ${ }^{4}$ They confined their attention mostly to cylindrical crystals whose radius equals their length. In view

*This work was supported by the United States Atomic Energy Commission. 1 J. G. Campbelland A. J. F. Boyle, Australian J. Phys, 6, 171 (1953).

1 J. G. Campbell and A. J. F. Boyle, Australian J. Phys. 6, 1

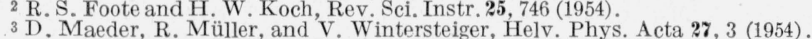

3 D. Maeder, R. Müller, and V. Wintersteiger, Helv. Phys. Acta 27, 3 (1954).
4 Maeder et al. also attempted a calculation for an "infinitely large" crystal, i.e., for a semi-infinite scattering medium, by considering the first two interactions exactly, and estimating the effect of subsequent interactions. As we shall show In section 4.1, this procedure was inaccurate (except at low energies) so that it does not provide a good basis for extrapolating their results from small to large crystals. of the complexity of their "orders of scattering" calculation and of the approximations whose consequences cannot be easily surveyed, a direct recalculation of their results seemed of value. Moreover, one would also like to know more about the influence of the crystal shape on the response function.

Monte Carlo calculations have been made of the response functions of cylindrical crystals of diverse shapes ranging in size from small to very large for radiation incident with energies of $0.279,0.661,1.17$, $1.33,2.62$, and $4.45 \mathrm{Mev}$.

The absorption and Compton scattering of photons in the crystals were calculated by random sampling, the computations being carried out on the SEAC. Approximate analytical corrections were applied to the Monte Carlo results to account for the escape of secondary bremsstrahlung and annihilation radiation from the crystals. $^{5}$ These corrections were quite small at 1.17 and $1.33 \mathrm{Mev}$, but appreciable at 2.62 and $4.45 \mathrm{Mev}$. Because of the approximate nature of the corrections, the most important and accurate parts of this work are those pertaining to the four lowest energies where the corrections were negligible. Most of the results are for collimated sources, while some sample calculations pertain to broad beams, point-isotropic sources, and off-axis collimated sources.

The main results of this investigation are: (1) The confirmation and expansion of the results of Maeder et al. for small crystals; (2) the extension to large crystals in which multiple interactions are important and to higher energies; (3) last but not least, the creation of a SEAC code by means of which response functions for source and crystal geometries peculiar to a given experimental situation can be readily computed with a modicum of effort.

5 One of the reasons for this approximate procedure was the temporary non availability of SEAC during the latter part of this investigation. It would availability of SEAC during the latter part of this investigation. It would have been better to carry through the entire calculation on the automatic com-
puter. This is particularly easy for quanta resulting from the annihilation of puter. This is particularly easy for quanta resulting from the annihilation of positrons produced in a first interaction of the primary photon. The source distributions of these photons within the crystal can easily be calculated, and their subsequent history traced by means of the existing SEAC code. We hope secondary radiation, and to extend the calculations above $4.45 \mathrm{Mev}$. In the meantime, there was some merit in reporting the present results for 2.62 and 4.45 $\mathrm{Mev}$, since calculations in this energy range have not been reported previously. 


\section{Method of Calculation}

\subsection{Formulation of the Problem}

The response function can be expressed in the form

$$
R\left(E, E^{\prime \prime}\right)=\int_{0}^{E} L\left(E, E^{\prime}\right) g\left(E^{\prime}, E^{\prime \prime}\right) d E^{\prime}
$$

where $L\left(E, E^{\prime}\right) d E^{\prime}$ is the probability that a photon of initial energy $E$ will deliver to the crystal - as the result of one or more interactions - an amount of energy between $E^{\prime}$ and $E^{\prime}+d E^{\prime}$; and $g\left(E^{\prime}, E^{\prime \prime}\right) d E^{\prime \prime}$ is the probability that upon delivery of energy $E^{\prime}$ to the crystal, the light output as amplified by the attached photomultiplier will indicate an apparent energy (pulse height) between $E^{\prime \prime}$ and $E^{\prime \prime}+d E^{\prime \prime}$.

It is an experimental fact that

$g\left(E^{\prime}, E^{\prime \prime}\right)=\left(2 \pi \alpha E^{\prime}\right)^{-1 / 2} \exp \left\{-\left(E^{\prime}-E^{\prime \prime}\right)^{2} / 2 \alpha E^{\prime}\right\}$.

The value of the parameter $\alpha$ depends on the physical characteristics of the crystal-photomultiplier system. We shall consider this parameter as determined experimentally, and concentrate on the calculation of the loss function $L\left(E, E^{\prime}\right)$.

To clarify the physical picture, it is useful to break the loss function up into a number of component parts, writing

$$
L\left(E, E^{\prime}\right)=Y(E)\left\{K\left(E, E^{\prime}\right)+p(E) \delta\left(E-E^{\prime}\right)\right\} .
$$

$Y(E)$ is the efficiency, i. e., the probability that a photon incident with energy $E$ will have at least one interaction (scattering or absorption) in the crystal. For a collimated source

$$
Y(E)=1-\exp [-\mu(E) L]
$$

where $L$ is the length of the crystal, and $\mu(E)$ the narrow beam linear absorption coefficient.

$K\left(E, E^{\prime}\right) d E^{\prime}$ is the probability that an interacting photon will emerge from the crystal after one or more Compton scatterings, leaving behind an amount of energy between $E^{\prime}$ and $E^{\prime \prime}+d E^{\prime}$. The function $p(E)$ represents the probability that an interacting photon will be absorbed (possibly after a number of Compton scatterings), while the delta function $\delta\left(E-E^{\prime}\right)$ indicates that the entire energy $E$ is delivered to the crystal in a photon history terminating in absorption. We note the normalization

$$
\int_{0}^{E} d E^{\prime} K\left(E, E^{\prime}\right)+p(E)=1 .
$$

For a wide range of conditions the shape of the loss function depends largely on the ratio of the numbers of absorbed and emergent photons, and only rather insensitively on $K\left(E, E^{\prime}\right)$. In a rough approxima- tion, the shape can therefore be characterized by $p(E)$, which-following Maeder-we shall call the photofraction. In experimental terms,

$$
p(E)=\frac{\begin{array}{c}
\text { area under the "photopeak" of the pulse } \\
\text { height distribution }
\end{array}}{\text { area under the entire pulse height distribution }} \text {. }
$$

Knowledge of the photofraction is useful in other connections. Thus if one wants to use a crystal for counting photons in the presence of a high background, it may be desirable to count only the large pulses resulting from complete photon absorption. The required "photoefficiency" of the crystal is $Y(E) p(E)$. Counting of photons in the "photopeak" only is particularly useful for disentangling complex spectra containing several lines. ${ }^{6}$

\subsection{Random Sampling}

For the calculation of the loss function, a direct stochastic analog method paralleling the physical processes in all respects, has been chosen. Scintillation crystals are very efficient detectors; hence the efficiency of an analog Monte Carlo calculation is also high, and the computational cost of statistical and analytical refinements would be out of proportion to the possible gain in efficiency.

The calculation proceeds along the following lines. We start a photon at a specified position on the crystal surface with specified energy and direction. The position of the first interaction is determined as if the photon moved in an infinite scattering medium; the distance traveled to the point of interaction is distributed exponentially, the mean of the distribution being the inverse of the total linear narrow beam absorption coefficient. If the interaction occurred outside the region occupied by the crystal, the photon is recorded as having had no interaction, and another photon history is begun. If the interaction is inside, we determine by random sampling whether it is an absorption or Compton scattering, the relative probabilities for these contingencies being proportional to the respective cross sections. In case of an absorption, the photon history is terminated, and another begun. In case of a scattering, the new energy and direction of the photon after the collision are sampled from the Klein-Nishina distribution, whereupon the location of the next interaction is sampled in the same manner as before. If this interaction occurs outside the crystal, the photon is considered to have escaped, and the energy left behind in the crystal is recorded. If the interaction was inside, we proceed as before, deciding whether it was an absorption or scattering, etc. The procedure is repeated until the photon has either left the crystal, or has reached an energy

\footnotetext{
${ }^{\circ}$ W. E. Kreger, Phys. Rev. 95, 1554 (1954); see also footnote 3.
} 
lower than $50 \mathrm{kev}$, in which case the photon is considered as absorbed. The bias introduced by this cutoff is negligible because of the steep rise of the photoelectric absorption cross section for $\mathrm{NaI}$ at energies below $50 \mathrm{kev}$.

The detailed equations for carrying out the sampling procedure have been presented elsewhere, together with an account of the numerical manipulation required to adapt the various cross sections to computation on a high-speed computer. ${ }^{7}$

The required absorption coefficients were obtained from the tabulation of $\mathrm{G}$. White. ${ }^{8}$

\subsection{Machine Computation}

In the SEAC program, the calculation was set up for the simultaneous consideration of nine crystals of different sizes. For the sake of convenience, these sizes were chosen so that the crystals formed a nested sequence, each crystal containing completely the next smaller one. When a photon in the random sampling experiment escaped from a crystal, the history was continued for all crystals of larger size. This procedure resulted in great economy of computing time. ${ }^{9}$ Beyond that, it increases the statistical efficiency of comparisons of the response functions of crystals of different shapes.

The output of a SEAC calculation consists of the following information for each of nine crystals:

a. The efficiency $Y(E)$. This information is in a sense redundant since it can also be easily obtained by a direct analytical calculation (eq (4)). But a comparison between analytical and Monte Carlo efficiencies provides a useful check that the computer operation was error-free.

b. The spectrum (in histogram form) of the energy packets supplied to the crystal by photons which eventually escape:

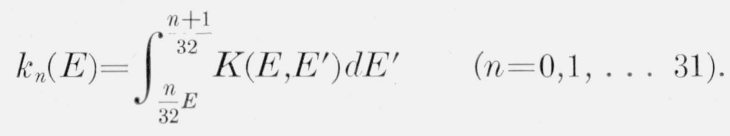

c. The photofraction $p(E)$.

It was convenient to generate and process photon histories in groups of 1,C00. For photons with an initial energy of $660 \mathrm{kev}$, this took about 35 to $40 \mathrm{~min}$ of SEAC time; for higher energies the running time was a little longer.

\subsection{Escape of Secondary Radiation}

In the Monte Carlo calculations it was assumed that the entire energy released in the successive scatterings or final absorption of the incident photons

\footnotetext{
${ }^{7}$ M. Berger, J. Research NBS 56, 343 (1956) RP2640.

$8 \mathrm{G}$. White (private communication).

$\checkmark$ There is another obvious way of increasing the efficiency of the computation, namely, by treating the first interaction analytically, and sampling only subsenamely, by treating the first interaction analytically, and sampling only subse-
quent interactions. This procedure avoids photon histories which result in no interaction at all, and thus contribute only information on the efficiency (which
}

remained in the crystal. Actually this is the case only at low energies. At energies above the threshold for pair production, annihilation quanta may escape from the crystal; additional energy can be lost in the form of bremsstrahlung. In the energy region of interest (up to $4.45 \mathrm{Mev}$ ) the effect of bremsstrahlung was found to be minor compared to that of annihilation radiation. Not only is the latter greater quantitatively, but it also affects the shape of the response function in a more distinct fashion, giving rise to two additional peaks corresponding to the complete escape of 1 or 2 annihilation quanta subsequent to the absorption of a primary photon.

The appropriate corrections were made according to the approximate procedure outlined in the appendix. The photofraction $p(E)$ was corrected both for annihilation radiation and bremsstrahlung. In order to avoid excessive computation, the detailed shape of the response function was corrected only for annihilation radiation, and an adjustment for the effects of bremsstrahlung was made by a subsequent renormalization of the function $K\left(E, E^{\prime}\right)$.

\subsection{Gaussian Broadening of the Response Function}

It follows from eq (1), (2), (3), and (4) that the response function

$$
\begin{aligned}
& R\left(E, E^{\prime \prime}\right)=\frac{Y, E)}{\sqrt{2 \pi \alpha E}} \\
& \int_{0}^{E} K\left(E, E^{\prime}\right) \frac{1}{\sqrt{E^{\prime}}} \exp \left\{-\left(E^{\prime \prime}\right.\right. \\
& \left.\left.-E^{\prime}\right)^{2} / 2 \alpha E^{\prime}\right\} d E^{\prime} \\
& +\frac{Y(E) p(E)}{\sqrt{2 \pi \alpha E}} \exp \left\{-\left(E^{\prime \prime}-E\right)^{2} / 2 \alpha E\right\} \\
& =H\left(\underset{\text { (tail) }}{\left.E^{\prime \prime}\right)}+\underset{\text { (photopeak) }}{G\left(E, E^{\prime \prime}\right)}\right.
\end{aligned}
$$

For numerical applications the parameter $\alpha$ was chosen to be $0.00146 E(\mathrm{Mev})$, corresponding to a width at half-maximum $W=0.09 \sqrt{E}$. This is a typical value in close agreement with experimental data of Foote and Koch (see footnote 2).

In the evaluation of $H\left(E, E^{\prime \prime}\right)$ one must take into account that $K\left(E, E^{\prime}\right)$ is available only in histogram form

$$
k_{n}=\int_{\frac{n}{32} E}^{\frac{n+1}{32} E} K\left(E, E^{\prime}\right) d E^{\prime}, \quad n=0,1, \ldots 31 .
$$

in any case can be determined analy tically), but not the shape of the loss function. This procedure leads to complications when one tries to consider several crystals simultaneously, and it was decided that-on balance-the possible gain in efficiency was not sufficient to compensate for the increased amount of manipulaton and computing time. 
It is assumed that the $k_{n}$ 's have been corrected if necessary, for escape of secondary radiation. We consider them to form a row matrix $k(E)=\left(k_{0} k_{1} \ldots\right.$ $\left.k_{31}\right)$. We similarly replace $H\left(E, E^{\prime \prime}\right)$ by a row matrix. $h(E)=\left(h_{0} h_{1} \ldots h_{31}\right)$, where $h_{n}(E)=\int_{\frac{n}{32} E}^{\frac{n+1}{32} E} H\left(E, E^{\prime}\right) d E^{\prime}$.

The convolution of $K\left(E, E^{\prime}\right)$ with a Gaussian is replaced by a matrix multiplication:

$$
h=T k,
$$

where the matrix element $T_{n m}$ (a kind of transition probability) is given by

$$
\left.\begin{array}{rl}
T_{n m}= & \int_{0}^{1} d t \frac{1}{\sqrt{2 \pi \alpha \frac{n+t}{32}}} \cdot \\
& \int_{\frac{m}{32} E}^{\frac{m+1}{32} E} d E^{\prime} \exp \left\{-\left(E^{\prime}\right\}\right. \\
& \left.\left.-\frac{n+t}{32} E\right)^{2} / 2 \alpha\left(\frac{n+t}{32}\right) E\right\}
\end{array}\right\}
$$

The exact location of a pulse within an interval of size $E / 32$ is not known from the Monte Carlo calculation. We assume a uniform distribution, which is the reason for the average with respect to $t$ in (9).

\section{Results}

\subsection{Efficiency and Photofraction for Collimated Radiation ${ }^{10}$}

Crystals of such sizes as are either readily available commercially, or are in use in this laboratory, were selected for computation. In table 1 , the crystal efficiencies are listed for collimated radiation of various energies incident along the axis of the cylindrical crystals, computed according to eq (4). The figures in parentheses indicate the values that were obtained by the Monte Carlo calculations. The agreement is excellent.

In table 2, the photofraction $p(E)$ is given. This is the corrected value taking into account the escape of secondary radiation. The amount $\Delta p$ which had to be subtracted from the raw Monte Carlo result $p_{0}$ in order to make the correction is indicated in parentheses. The indicated errors are standard deviations computed as follows. The standard deviation of $p_{0}$ is

$$
\sigma_{0}=\left[\frac{p_{0}\left(1-p_{0}\right)}{Y N}\right]^{1 / 2}
$$

where $N$ is the total number of photon histories contributing to the determination of $p_{0} . \quad(N$ is also shown in tables 1 and 2.) The correction $\Delta p$ was assumed to have a standard deviation (1/10) $\Delta p$. This is a nominal figure representing an "educated guess." The total standard deviation is

$$
\sigma=\left[\sigma_{0}^{2}+(1 / 10 \Delta p)^{2}\right]^{1 / 2} .
$$

${ }_{10}$ Collimated radiation means a narrow pencil of radiation incident along the

\begin{tabular}{|c|c|c|c|c|c|c|c|c|c|c|}
\hline Crystal No. & 1 & 2 & 3 & 4 & 5 & 6 & 7 & 8 & 9 & \multirow{4}{*}{$\begin{array}{l}\text { No. of } \\
\text { histories } \\
N\end{array}$} \\
\hline Radius (in.) & 2.5 & 2.5 & 2.5 & 0.875 & 0.75 & 0.75 & 0.75 & 0.5 & 0.25 & \\
\hline \multirow[t]{2}{*}{ Length (in.) } & 9.0 & 8.0 & 4. 0 & 2. 0 & 2. 0 & 1.5 & 1.0 & 1. 0 & 0.5 & \\
\hline & \multicolumn{9}{|c|}{ Efficiency $Y(E)$} & \\
\hline \multicolumn{11}{|l|}{$\underset{(\text { Mev })}{\text { Energy } E}$} \\
\hline 0.279 & $\left\{\begin{array}{c}1.000 \\
(1.000)\end{array}\right.$ & $\begin{array}{c}1.000 \\
(1.000)\end{array}$ & $\begin{array}{l}0.998 \\
(.999)\end{array}$ & $\begin{array}{l}0.958 \\
(.957)\end{array}$ & $\begin{array}{c}0.958 \\
(.957)\end{array}$ & $\begin{array}{l}0.908 \\
(.903)\end{array}$ & $\begin{array}{l}0.796 \\
(.798)\end{array}$ & $\begin{array}{l}0.796 \\
(.798)\end{array}$ & $\begin{array}{l}0.548 \\
(.546)\end{array}$ & 6,000 \\
\hline .661 & $\left\{\begin{array}{l}0.998 \\
(.998)\end{array}\right.$ & $\begin{array}{l}0.996 \\
(.996)\end{array}$ & $\begin{array}{l}.936 \\
(.938)\end{array}$ & $\begin{array}{l}.747 \\
(.748)\end{array}$ & $\begin{array}{l}.747 \\
(.748)\end{array}$ & $\begin{array}{l}.643 \\
(.644)\end{array}$ & $\begin{array}{l}.497 \\
(.497)\end{array}$ & $\begin{array}{l}.497 \\
(.497)\end{array}$ & $\begin{array}{l}.290 \\
(.295)\end{array}$ & 10,000 \\
\hline 1.17 & $\left\{\begin{array}{c}.988 \\
(.987)\end{array}\right.$ & $\begin{array}{l}.981 \\
(.979)\end{array}$ & $\begin{array}{l}.861 \\
(.859)\end{array}$ & $\begin{array}{l}.627 \\
(.635)\end{array}$ & $\begin{array}{l}.627 \\
(.635)\end{array}$ & $\begin{array}{r}.522 \\
(.529)\end{array}$ & $\begin{array}{l}.389 \\
(.395)\end{array}$ & $\begin{array}{l}.389 \\
(.395)\end{array}$ & $\begin{array}{l}.218 \\
(.223)\end{array}$ & 5,000 \\
\hline 1. 33 & $\left\{\begin{array}{c}.984 \\
(.986)\end{array}\right.$ & $\begin{array}{l}.975 \\
(.977)\end{array}$ & $\begin{array}{l}.841 \\
(.833)\end{array}$ & $\begin{array}{l}.602 \\
(.599)\end{array}$ & $\begin{array}{l}.602 \\
(.599)\end{array}$ & $\begin{array}{l}.499 \\
(.495)\end{array}$ & $\begin{array}{l}.369 \\
(.363)\end{array}$ & $\begin{array}{l}.369 \\
(.363)\end{array}$ & $\begin{array}{l}.206 \\
(.191)\end{array}$ & 5,000 \\
\hline 2. 62 & $\left\{\begin{array}{c}.958 \\
(.964)\end{array}\right.$ & $\begin{array}{l}.940 \\
(.946)\end{array}$ & $\begin{array}{l}.755 \\
(.760)\end{array}$ & $\begin{array}{l}.504 \\
(.507)\end{array}$ & $\begin{array}{l}.504 \\
(.507)\end{array}$ & $\begin{array}{l}.410 \\
(.409)\end{array}$ & $\begin{array}{l}.296 \\
(.292)\end{array}$ & $\begin{array}{l}.296 \\
(.292)\end{array}$ & $\begin{array}{l}161 \\
(.164)\end{array}$ & 6,000 \\
\hline 4. 45 & $\left\{\begin{array}{c}.950 \\
(.947)\end{array}\right.$ & $\begin{array}{l}.930 \\
(.927)\end{array}$ & $\begin{array}{l}.735 \\
(.741)\end{array}$ & $\begin{array}{l}.486 \\
(.484)\end{array}$ & $\begin{array}{l}.486 \\
(.484)\end{array}$ & $\begin{array}{l}.393 \\
(.398)\end{array}$ & $\begin{array}{l}.283 \\
(.282)\end{array}$ & $\begin{array}{l}.283 \\
(.282)\end{array}$ & $\begin{array}{l}.153 \\
(.153)\end{array}$ & 5,000 \\
\hline
\end{tabular}
crystal axis.

Table 1. Crystal efficiency

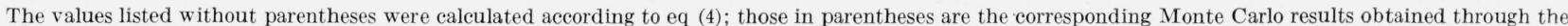
analysis of the indicated number of photon histories. 
TABLE 2. Photofraction $p(E)$

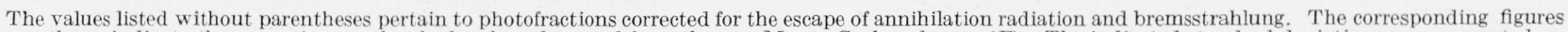

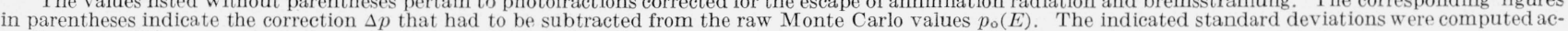
cording to eq (11).

\begin{tabular}{|c|c|c|c|c|c|c|c|c|c|c|}
\hline Crystal No. & 1 & 2 & 3 & 4 & 5 & 6 & 7 & 8 & 9 & \multirow{4}{*}{$\begin{array}{c}\text { No. of } \\
\text { histories } \\
N\end{array}$} \\
\hline Radius (in.) & 2.5 & 2. 5 & 2.5 & 0.875 & 0.75 & 0.75 & 0.75 & 0.5 & 0.25 & \\
\hline \multirow[t]{2}{*}{ Length (in.) } & 9.0 & 8.0 & 4.0 & 2. 0 & 2.0 & 1.5 & 1. 0 & 1.0 & 0.5 & \\
\hline & \multicolumn{9}{|c|}{ Photofraction $p(E)$} & \\
\hline \multicolumn{11}{|l|}{$\underset{\text { (Mer) }}{\operatorname{Energy}} E$} \\
\hline 0.279 & $\left\{\begin{array}{r}0.973 \\
\pm .002\end{array}\right.$ & $\begin{array}{r}0.973 \\
\pm .002\end{array}$ & $\begin{array}{r}0.971 \\
\pm .002\end{array}$ & $\begin{array}{r}0.915 \\
\pm .004\end{array}$ & $\begin{array}{r}0.900 \\
\pm .004\end{array}$ & $\begin{array}{r}0.882 \\
\pm .004\end{array}$ & $\begin{array}{r}0.843 \\
\pm .005\end{array}$ & $\begin{array}{r}0.814 \\
\pm .006\end{array}$ & $\begin{array}{r}0.711 \\
\pm .008\end{array}$ & 6,000 \\
\hline .661 & $\left\{\begin{array}{r}.887 \\
\pm .003\end{array}\right.$ & $\begin{array}{r}.884 \\
\pm .003\end{array}$ & $\begin{array}{r}.821 \\
\pm .004\end{array}$ & $\begin{array}{r}.542 \\
\pm .005\end{array}$ & $\begin{array}{r}.508 \\
\pm .006\end{array}$ & $\begin{array}{r}.481 \\
\pm .006\end{array}$ & $\begin{array}{r}.442 \\
\pm .007\end{array}$ & $\begin{array}{r}.377 \\
\pm .007\end{array}$ & $\begin{array}{r}.243 \\
\pm .008\end{array}$ & 10,000 \\
\hline 1. 17 & $\left\{\begin{array}{r}.778 \\
\pm .006 \\
(.002)\end{array}\right.$ & $\begin{array}{r}.775 \\
+.006 \\
(.002)\end{array}$ & $\begin{array}{r}.682 \\
\pm .007 \\
(.002)\end{array}$ & $\begin{array}{r}.368 \\
\pm .008 \\
(.003)\end{array}$ & $\begin{array}{r}.343 \\
\pm .008 \\
(.003)\end{array}$ & $\begin{array}{r}.315 \\
\pm .009 \\
(.005)\end{array}$ & $\begin{array}{r}.282 \\
\pm .010 \\
(.005)\end{array}$ & $\begin{array}{r}.235 \\
\pm .010 \\
(.005)\end{array}$ & $\begin{array}{r}.140 \\
+.011 \\
(.003)\end{array}$ & 5,000 \\
\hline 1. 33 & $\left\{\begin{array}{r}.767 \\
\pm .006 \\
(.003)\end{array}\right.$ & $\begin{array}{r}.758 \\
\pm .006 \\
(.004)\end{array}$ & $\begin{array}{r}.667 \\
\pm .007 \\
(.005)\end{array}$ & $\begin{array}{r}.351 \\
\pm .009 \\
(.009)\end{array}$ & $\begin{array}{r}.325 \\
\pm .009 \\
(.009)\end{array}$ & $\begin{array}{r}.303 \\
\pm .009 \\
(.010)\end{array}$ & $\begin{array}{r}.272 \\
\pm .010 \\
(.013)\end{array}$ & $\begin{array}{r}.224 \\
\pm .010 \\
(.013)\end{array}$ & $\begin{array}{r}.120 \\
\pm .011 \\
(.011)\end{array}$ & 5,000 \\
\hline 2.62 & $\left\{\begin{array}{r}.653 \\
\pm .008 \\
(.051)\end{array}\right.$ & $\begin{array}{r}.643 \\
\pm .009 \\
(.056)\end{array}$ & $\begin{array}{r}.531 \\
\pm .010 \\
(.069)\end{array}$ & $\begin{array}{r}.243 \\
+.014 \\
(.117)\end{array}$ & $\begin{array}{r}.226 \\
\pm .015 \\
(.122)\end{array}$ & $\begin{array}{r}.201 \\
\pm .016 \\
(.128)\end{array}$ & $\begin{array}{r}.175 \\
+.017 \\
(.135)\end{array}$ & $\begin{array}{r}.144 \\
+.017 \\
(.131)\end{array}$ & $\begin{aligned} & .0950 \\
& \pm .018 \\
&(.130)\end{aligned}$ & 6,000 \\
\hline 4.45 & $\left\{\begin{array}{r}.621 \\
\pm .015 \\
(.139)\end{array}\right.$ & $\begin{array}{r}.608 \\
\pm .015 \\
(.141)\end{array}$ & $\begin{array}{r}.491 \\
+.020 \\
(.184)\end{array}$ & $\begin{array}{r}.169 \\
\pm .033 \\
(.319)\end{array}$ & $\begin{array}{r}.157 \\
\pm .033 \\
(.319)\end{array}$ & $\begin{array}{r}.132 \\
\pm .035 \\
(.332)\end{array}$ & $\begin{array}{r}.119 \\
\pm .036 \\
(.331)\end{array}$ & $\begin{aligned} & .0826 \\
& \pm .037 \\
&(.347)\end{aligned}$ & $\begin{array}{r}.0890 \\
\pm .039 \\
(.349)\end{array}$ & 5,000 \\
\hline
\end{tabular}

\subsection{An Empirical Formula for the Photofraction}

It has already been noted by Maeder et al., that for small crystals at an energy of $0.511 \mathrm{Mev}$ the photofraction is a smooth function of $\sqrt{L R}$ where $L$ is the crystal length and $R$ the radius. The results of table 2 show that this also holds true for large crystals and in the entire energy range from 0.279 to $4.45 \mathrm{Mev}$.

Moreover, the dependence of the photofraction on the crystal size can be accurately represented by a simple empirical formula, under conditions where the correction due to the escape of secondary radiation is absent or small (i. e., at $0.279,0.661$, and 1.17 Mev for all the crystals, and at 1.33 for all but the smallest crystal considered.) $)^{11}$

The formula is:

$$
p(\sqrt{L R}, E)=A(E)-B(E) e^{-C(E) \sqrt{L R}}
$$

where $L$ and $R$ are the length and radius of the crystal. With the parameters $\mathrm{A}, \mathrm{B}$, and $\mathrm{C}$ listed in table 3 , formula (12) represents the values of $p(E)$ given in table 2 with an accuracy that is 1 to 2 percent in most cases, occasionally 3 to 4 percent, and in the worst cases 5 to 7 percent. These deviations are presumably due to the fact that $p(E)$ depends on $L$ and $R$ in a more complicated way than through $\sqrt{L R}$.

11iAt higher energies a simple expression of the type (12) does not appear to work well. In any case the high-energy results are not precise enough to make fitting by a formula worthwhile.
TABle 3. Parameters of the empirical formula, eq (12), for the photofraction

\begin{tabular}{|c|r|r|r|}
\hline$E$ & $\mathrm{~A}(E)$ & $\mathrm{B}(E)$ & $\mathrm{C}(E)$ \\
\cline { 1 - 1 } & & & \\
\cline { 1 - 1 } Mev & & & \\
0.279 & 0.973 & 0.514 & 0.658 \\
.661 & .940 & .841 & .219 \\
1.17 & .980 & .934 & .124 \\
1.33 & .985 & .942 & .120 \\
\hline
\end{tabular}

Formula (12) also predicts the correct number albedo for a semi-infinite medium. When $L \rightarrow \infty$ and $R \rightarrow \infty$

$$
p(\infty, E)=\mathrm{A}(E)=1-\text { number albedo. }
$$

When we extrapolate in the other direction $(L \rightarrow 0$ and $R \rightarrow 0)$

$$
p(0, E)=\mathrm{A}(E)-\mathrm{B}(E)=\mu_{\mathrm{PH}} / \mu
$$

where $\mu_{\mathrm{PH}} / \mu$ is the ratio of the photoelectric to the total attenuation coefficient. Thus, of the three parameters A, B, and C, two are fixed by (13) and (14), and only C, a quantity with the dimensions of an attenuation coefficient, is adjustable. This makes it rather remarkable that a simple formula like (12) works. 


\subsection{Shape of the Response Function for Collimated Radiation}

The response functions for all the crystals listed in tables 1 and 2 (except No. 2) are shown in figures $1, \mathrm{a}$ and $1, \mathrm{~b}$ for incident energies of $0.279,0.661$, $1.17,1.33,2.62$, and $4.45 \mathrm{Mev}$. For the sake of clarity of presentation we have plotted only the response function exclusive of the photopeak, i. e., the function $H\left(E, E^{\prime}\right)$ as defined in eq (7). The photopeaks are indicated by arrows.

'The Gaussian broadening has been calculated according to the prescription given in section 2.5. It should be mentioned that the shape of the response function is rather insensitive to the magnitude of $\alpha$. The results are presented in histogram form, the range between the energy of incidence $E$, and zero energy being covered by 32 intervals of equal size.

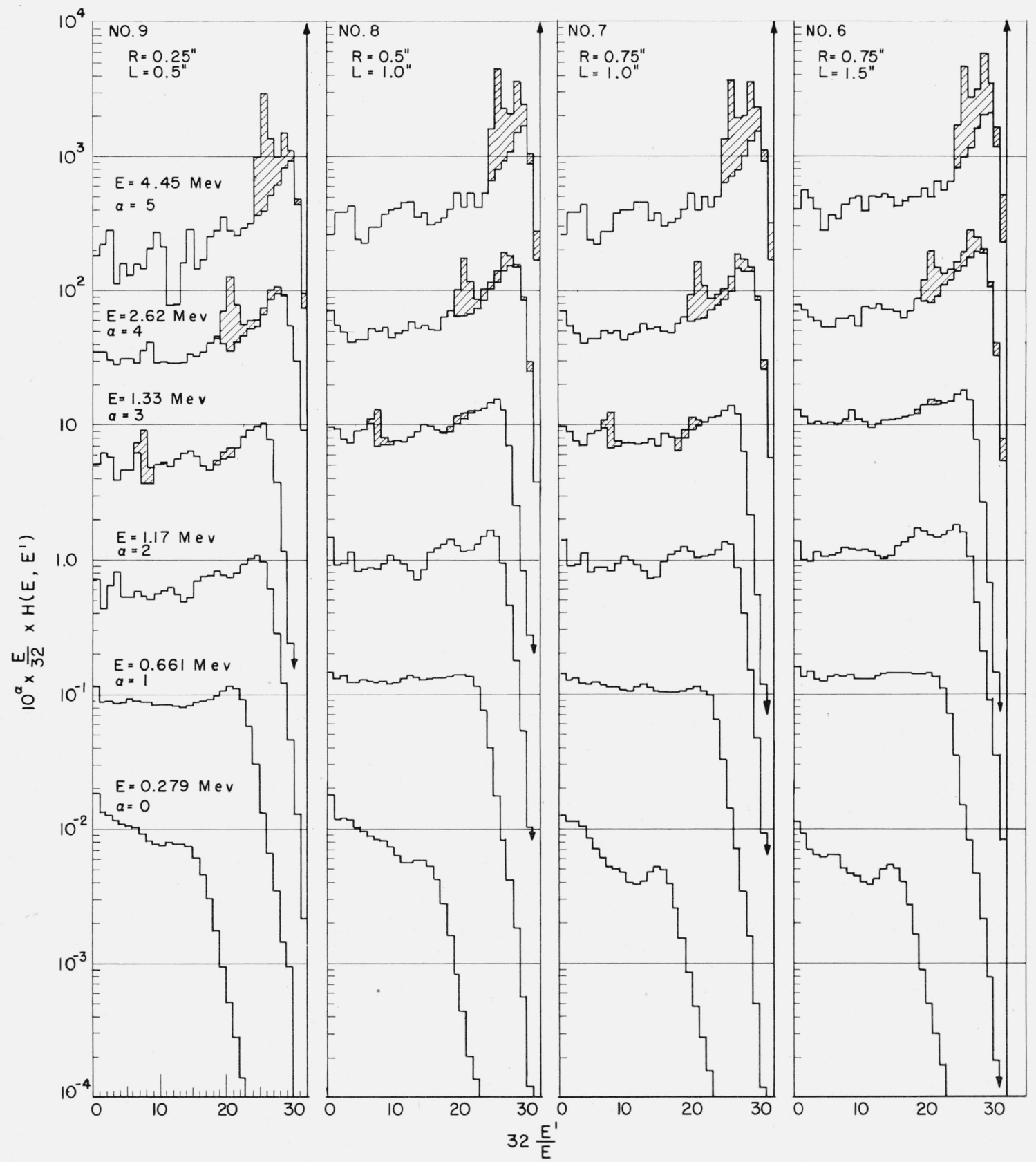

FIGURE 1. Response functions of $\mathrm{NaI}(\mathrm{Tl})$ scintillation crystals for collimated radiation incident along the crystal axis. a, Crystals No. 9, 8, 7, and 6 .

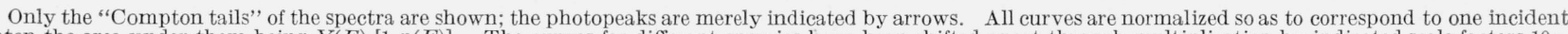

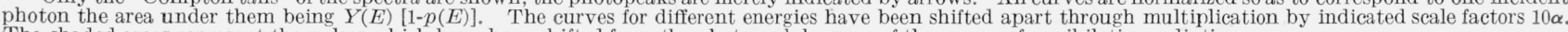
The shaded areas represent the pulses which have been shifted from the photopeak because of the escape of annihilation radiation. 
The histograms are normalized absolutely so that they correspond to one photon incident on a crystal. The area under each histogram is thus $Y(E)[1-p(E)] .^{12}$ In order to supply the missing photopeak, one need merely add a Gaussian with the desired standard deviation (cf. eq (7)) and multiplied by a weight factor $Y(E) p(E) ; Y(E)$ and $p(E)$ can be found in tables 1 and 2 .

The shaded parts of the histograms indicate the

12 To separate the histograms for different energies, the ordinates were multiplied by indicated scale factors $10 \alpha$. contribution to the scintillation spectrum due to the escape of secondary radiation. In other words, they represent pulses which would have ended up in the photopeak if the absorptions had not been spurious. It can be seen that at sufficiently high source energies the response functions have two peaks at energies $\mathrm{mc}^{2}$ and $2 \mathrm{mc}^{2}$ below the incident energy $E$, which are associated with the escape of 1 or 2 annihilation quanta.

It can be seen that the shapes of the response functions for various crvstal sizes, and even for different energies, are on the whole rather similar.

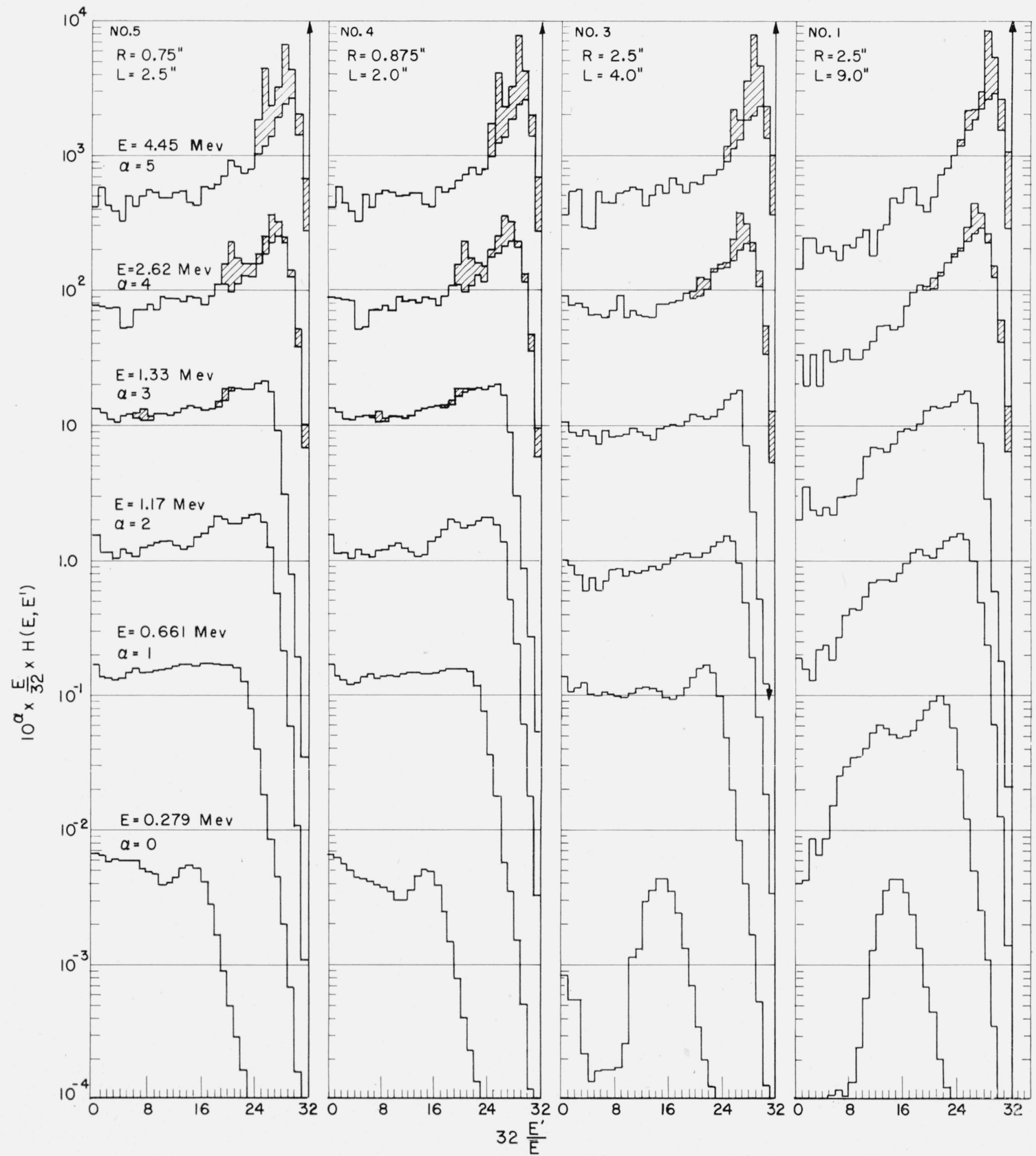

FIGURE 1. Response functions of $\mathrm{NaI}(\mathrm{Tl})$ scintillation crystals for collimated radiation incident along the crystal axis- $\mathrm{Con}$. b, Crystals No. 5, 4, 3, and 1 . 
The statistical fluctuations of the Monte Carlo calculations, as evidenced by the irregularities of the histograms, are much worse for high than for low incident energies. It is noticeable that quite similar local irregularities occur for histograms pertaining to the same energy but different crystals. This phenomenon is due to the use of the same groups of photon histories for all the crystals.

\subsection{Other Source Geometries}

Monte Carlo calculations of the response function for any desired source geometry can easily be accomplished through the proper choice of the initial conditions, photon positions and directions, in the random sampling process. Sample results of such calculations for $0.661 \mathrm{Mev}$ radiation are presented in figures 2,3 , and 4 .

\section{a. Broad-Beam Radiation}

In figure 2 the ratio $p_{\mathrm{BB}} / p$ is plotted against $r / R$, where $p_{\mathrm{BB}}$ is the photofraction for a broad cylindrical

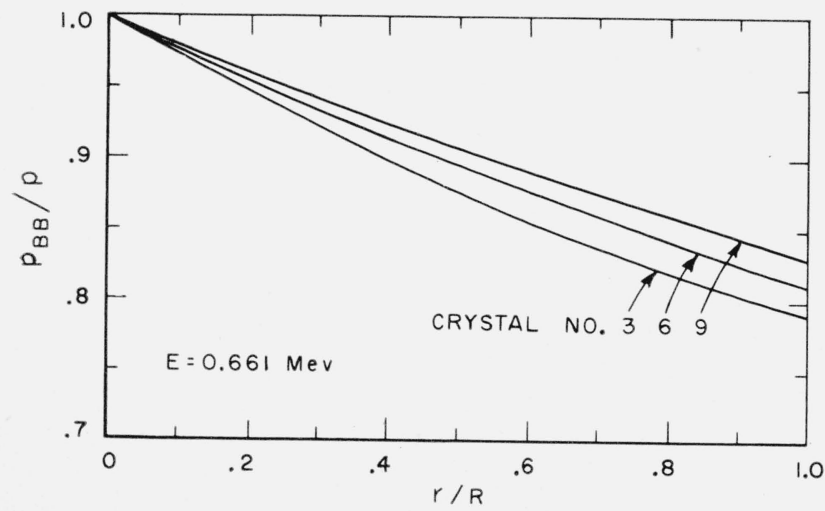

Figure 2. Comparison of the photofractions for broad beams, and for collimated radiation incident along the crystal axis.

The ratio $p_{\mathrm{B} \mathrm{B} / p}$ is plotted as a function of $r / R$, where $r$ is the radius of the broad beam, and $R$ that of the crystal. The source energy is $0.661 \mathrm{Mev}$.

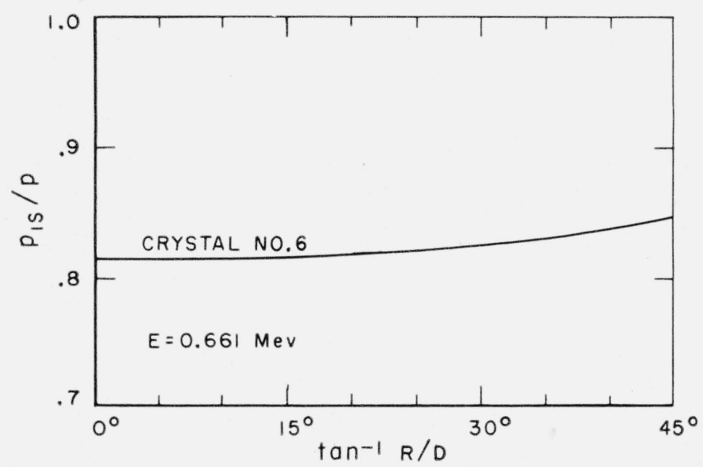

FIguRE 3. Comparison of the photofractions for point-isotropic sources, and for collimated radiation incident along the crystal axis.

The ratio $p_{I S} / p$ is plotted as a function of $\tan ^{-1}(D / R)$, where $D$ is the distance from the crystal to the point source (located on the crystal axis), and $R$ is the crystal radius. beam with cross-sectional radius $r, p$ is the photofraction for a collimated source, and $R$ is the crystal radius. Three curves are shown, for crystals No. 3 , large; No. 6, medium-sized; and No. 9, small.

\section{b. Point-Isotropic Source}

In figure 3 , the ratio $p_{\text {IS }} / p$ is plotted against $\tan ^{-1} R / D$ where $p_{\text {Is }}$ is the photofraction for a pointisotropic source located at a distance $D$ from the crystal on the cylinder axis. The curve pertains to crystal No. 6. The end-point of this curve, for $\tan ^{-1} R / D=0^{\circ}$, was obtained from the corresponding broad-beam result.

\section{c. Off-Axis Collimated Beam}

In figure 4 , the shape of the response function is shown for collimated pencils of radiation incident at various distances $s$ from the crystal axis, for crystals No. 4 and No. 1. The normalization and other details are the same as in figure 1; the photofractions are also shown.

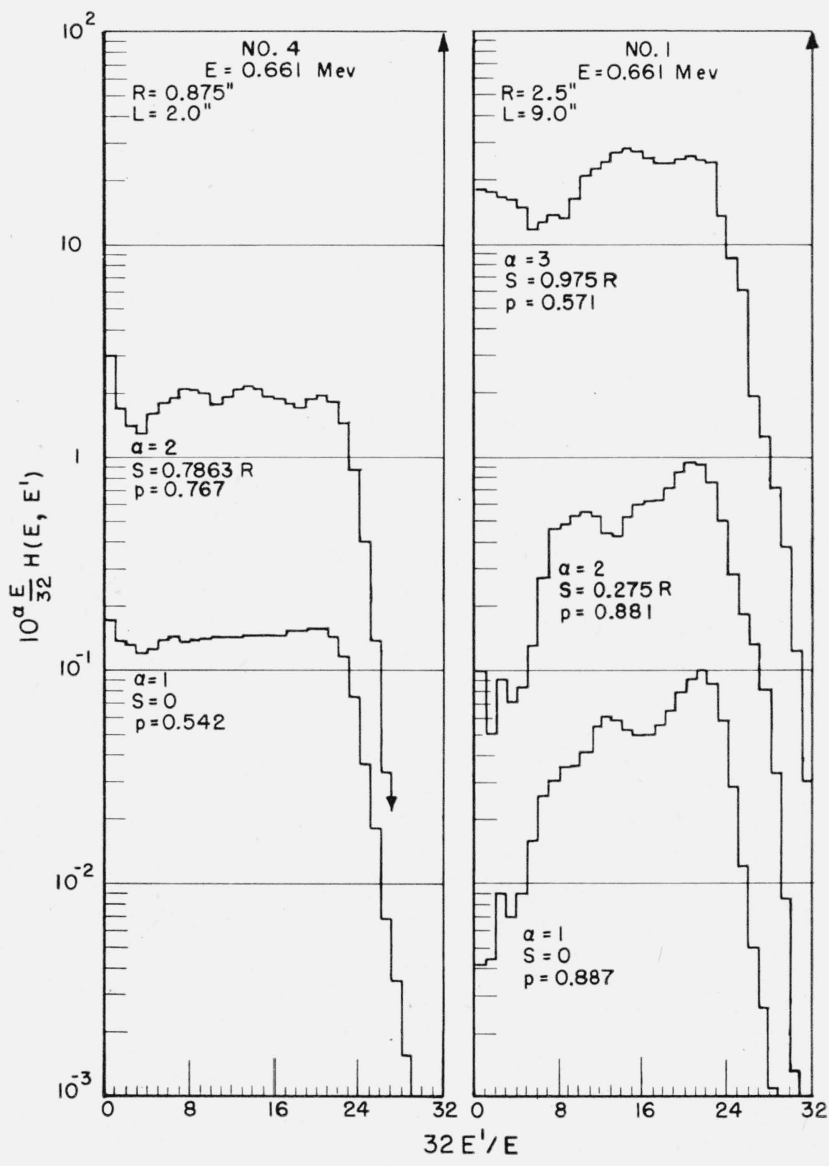

Figure 4. Response function of $\mathrm{NaI}(\mathrm{Tl})$ scintillation crystals for collimated radiation incident offcenter at a distance s from the crystal axis.

The curves are normalized so as to correspond to one incident photon, the area under them being $Y(E)[1-p(E)]$. The curves for different values of $s$ have been shifted apart through multiplication by indicated scale factors $10 \alpha$. Only the "Compton tails" of the spectra are shiown; the arrows, and the values of the photofractions are shown beside the correated arrows, and the values of the photofractions are shown beside the corresponding 


\section{Discussion}

\subsection{Comparison With Other Calculations}

Our results for the value of the photofraction for collimated radiation incident on small crystals are in good agreement with those obtained by Maeder et al. For example, at an energy of $0.661 \mathrm{Mev}$ one finds:

\begin{tabular}{|c|c|c|c|c|c|c|}
\hline & \multicolumn{6}{|c|}{ Crystal number } \\
\hline & 9 & 8 & 7 & 6 & 5 & 4 \\
\hline $\begin{array}{l}p(\text { Maeder }) \\
p(\text { table 2) }\end{array}$ & $\begin{array}{r}0.246 \\
.243\end{array}$ & $\begin{array}{r}0.368 \\
.377\end{array}$ & $\begin{array}{r}0.418 \\
.442\end{array}$ & $\begin{array}{r}0.471 \\
.481\end{array}$ & $\begin{array}{r}0.507 \\
.508\end{array}$ & $\begin{array}{r}0.546 \\
.542\end{array}$ \\
\hline
\end{tabular}

At $0.279 \mathrm{Mev}$ and $1.17 \mathrm{Mev}$ the agreement is almost as good (with the exception of a 15 percent discrepancy for the smallest crystal (No. 9) at $1.17 \mathrm{Mev})$.

No other results comparable to our photofractions for large crystals No. 1, 2, and 3 exist. Maeder et al. did, however, attempt to compute the photofraction for the limiting case of an infinitely large crystal (i. e., a semi-infinite medium), calculating the first two interactions of the incident photon exactly, and estimating the effect of the subsequent interactions. We have calculated the photofraction for this case by extrapolation based on the empirical formula (12) and arrive at the following comparison:

\begin{tabular}{|c|c|c|c|}
\hline \multirow{2}{*}{ Infinitely large crystal } & \multicolumn{3}{|c|}{ Energy (Mev) } \\
\hline & 0. 279 & 0.661 & 1. 17 \\
\hline $\begin{array}{l}p(\text { Monte Carlo }) \ldots \\
p(\text { Maeder })\end{array}$ & $\begin{array}{r}0.975 \\
.975\end{array}$ & $\begin{array}{r}0.940 \\
.876\end{array}$ & $\begin{array}{r}0.980 \\
.802\end{array}$ \\
\hline
\end{tabular}

The "orders of scattering" approach, in the limit of very large crystals, agrees with the more exact calculation only at $0.279 \mathrm{Mev}$, where multiple interactions are unlikely because of the large gamma-ray absorption cross section, but leads to a significant underestimate of the photofraction at higher energies.

Our albedo values $1-p$ are in good agreement with the results of a calculation by Hayward and Hubble. ${ }^{13}$ Note that the albedo is greater at $0.661 \mathrm{Mev}$ than at 0.279 or $1.17 \mathrm{Mev}$. There is also experimental evidence for the occurrence of this maximum at an intermediate energy. ${ }^{14}$

The pictures of the response function given by Maeder et al. are similar in appearance to ours, but not detailed enough for an accurate comparison in regard to shape.

\subsection{Comparison With Experimental Results}

For small crystals a detailed comparison with experiments has been made by Maeder et al., which

13 E. Hayward and J. Hubbell, Phys. Rev. 93, 955 (1954)

${ }_{14} \mathrm{H}$. W. Koch (private communication). can equally well be applied to our results. It indicates fair agreement between theory and experiment, but there is a consistent tendency for the experimental photofractions to be somewhat lower than the theoretical values. This discrepancy may be reduced in part if one takes into account energy losses due to electron escape from the crystal. ${ }^{15}$

Measurements of Foote and Koch (see footnote 2) indicate for collimated radiation incident on a large crystal ( $R=2.5$ in., $L=4$ in.) photofractions $p=0.8$ at $0.661 \mathrm{Mev}$, and 0.64 for cobalt-60 radiation, while the corresponding theoretical values are $p=0.821$, and 0.675 respectively. ${ }^{16}$

Further results for a large crystal $(R=2$ in., $L=4$ in.) have recently been reported by Kreger (see footnote 6 ). His incident radiation was a broad beam with cross-sectional radius $r=0.25 R$. Hence the comparison must be made for the broad-beam photofraction $p_{\mathrm{BB}}$. We have so far only calculated this correction at an energy of $0.661 \mathrm{Mev}$, but estimate that with an error of only a few percent the same fractional correction can also be applied at the other energies of Kreger's experiment.

\begin{tabular}{|l|c|c|c|c|c|c|}
\hline & \multicolumn{5}{|c|}{ Energy (Mev) } \\
\cline { 2 - 6 } & 0.279 & 0.661 & 1.17 & 1.32 & 2.62 \\
\hline$p$ (Kreger)__ & 0.93 & 0.725 & 0.57 & 0.54 & 0.47 \\
$p$ (present results)_ & $(.90)$ & .74 & $(.60)$ & $(.58)$ & $(.50)$ \\
\hline
\end{tabular}

The agreement is seen to be quite good at $0.661 \mathrm{Mev}$, where the exact value of the correction is known, and fairly good elsewhere.

Summing up the entire experimental evidence, both for small and large crystals, we can state the experimental photofraction is invariably below the theoretical values, by a smaller or greater amount. One is led to the conclusion that in the experiments there is always background radiation present (such as back-scattered radiation from the radiation source, and from the material surrounding the detector) which would be counted in the tail end of the pulseheight spectrum and would thus tend to depress the value of the photofraction.

The authors thank Dr. Evans Hayward for several stimulating and enlightening discussions, and Miss Mary Orr for her assistance with the hand computations.

\section{Appendix: Escape of Secondary Radiation}

\subsection{Effect on the Photofraction}

To estimate the effect of the escape of secondary radiation on the value of the photofraction $p$, we must determine the probability that an absorption is

15 K. Lidén and N. Starfelt, Arkiv Fysik $\boldsymbol{\gamma}, 428$ (1954). ${ }_{16}$ The photofraction for cobalt-60 radiation, $\bar{p}=\frac{Y(1.17) p(1.17)+Y(1.33) p(1.33)}{Y(1.17)+Y(1.33)}$. 
"spurious," i. e., that it results in, or is preceded by, the escape of at least one secondary photon from the crystal (with an energy greater than $50 \mathrm{kev}) .^{17}$

We shall ignore the displacement of charged particles in the crystal. $^{18}$ Bremsstrahlung and annihilation quanta are thus assumed to be emitted at the point of absorption of the primary photon. Moreover, the emission is assumed to be isotropic. First, attention is concentrated on the spurious absorptions which occur in the first interaction of the incident photon (i. e., prior to any Compton scatterings), and return later to the relatively unimportant spurious absorptions resulting from other interactions. Secondary radiation is therefore considered as originating from an exponentially distributed line source on the axis of the crystal (on the assumption that the incident radiation is collimated).

The following notation will be used for the various attenuation coefficients: $\mu_{\mathbf{P H}}=$ photoelectric absorption coefficient, $\mu_{\mathrm{p}}=$ pair production absorption coefficient, $\mu_{\mathrm{C}}=$ Compton scattering attenuation coefficient; $\mu=\mu_{\mathrm{PH}}+\mu_{\mathrm{p}}+\mu_{\mathrm{C}}=$ total attenuation coefficient.

Annitilation Radiation. If an absorption of a primary photon of energy $E$ results in pair formation and subsequent annihilation of the positron, the probability that at least one of the two annihilation quanta will escape from a cylindrical crystal (with radius $R$ and length $L$ ) is given, approximately, by the following expression:

$$
\begin{aligned}
p_{0}= & \frac{\mu(E)}{1-\exp [-\mu(E) L]} \int_{0}^{L} d x e^{-\mu(E) x} \\
& \int_{0}^{1} d \cos \theta\left\{1-\left(1-e^{-s_{1} \mu^{\prime}}\right)\left(1-e^{-s_{2} \mu^{\prime}}\right) p_{1}^{*} p_{2}^{*}\right\}
\end{aligned}
$$

where $\mu^{\prime}=\mu\left(\mathrm{mc}^{2}\right)$, and $s_{1}$ and $s_{2}$ are the distances traveled in the crystal by the two annihilation quanta (emitted in opposite directions).

If $0 \leq x \leq L / 2$

$$
\begin{array}{lll}
\cos \theta_{1} \leq \cos \theta \leq 1 & s_{1}=x / \cos \theta & s_{2}=(L-x) / \cos \theta \\
\cos \theta_{2} \leq \cos \theta \leq \cos \theta_{1} & s_{1}=x / \cos \theta & s_{2}=R / \sin \theta \\
0 \leq \cos \theta \leq \cos \theta_{2} & s_{1}=R / \sin \theta & s_{2}=R / \sin \theta
\end{array}
$$

If $L / 2 \leq x \leq L$

$\cos \theta_{2} \leq \cos \theta \leq 1 \quad s_{1}=x / \cos \theta \quad s_{2}=(L-x) / \cos \theta$

$\cos \theta_{1} \leq \cos \theta \leq \cos \theta_{2} \quad s_{1}=R / \sin \theta \quad s_{2}=(L-x) / \cos \theta$

$0 \leq \cos \theta \leq \cos \theta_{1} \quad s_{1}=R / \sin \theta \quad s_{2}=R / \sin \theta$

where

$$
\cos \theta_{1}=\left[1+\left(\frac{R}{L-x}\right)^{2}\right]^{-1 / 2}, \cos \theta_{2}=\left[1+\left(\frac{R}{x}\right)^{2}\right]^{-1 / 2} .
$$

17 The limit of $50 \mathrm{kev}$ was selected because the same low-energy cutoff was also used in the main Monte Carlo calculation.

${ }_{18}$ The ranges for $0.3,1.0$, and $4.0 \mathrm{Mev}$ electrons in NaI are approximately 0.02 , 0.17 , and $0.861 \mathrm{~cm}$, respectively. Hence only at the highest energy $(4.45 \mathrm{Mev})$ and for the two smallest of the crystals considered (No. 8 and 9 ) could the neglect of electron displacement lead to a significant overestimate of the photofraction. $p_{1}^{*}$ and $p_{2}^{*}$ are photofractions for the two annihilation quanta, which-we believe-can be accurately approximated by the photofraction $p$ for an energy of $0.511 \mathrm{Mev}$, as obtained by interpolation from table 2 .

Bremsstrahlung. The probability of escape of a bremsstrahlung quantum of energy $E^{\prime}$ can be expressed by a formula similar to (15):

$$
\begin{aligned}
P_{1}\left(E^{\prime}\right)= & \frac{\mu(E)}{1-\exp [-\mu(E) L]} \int_{0}^{L} d x e^{-\mu(E) x} \\
& \frac{1}{2} \int_{-1}^{1} d \cos \theta\left\{e^{-s_{\mu}\left(E^{\prime}\right)}+\left[1-e^{-\delta \mu\left(E^{\prime}\right)}\right]\left[1-p^{*}(E)\right]\right\}
\end{aligned}
$$

where we again set $p^{*}(E)=p(E)$, and the path length $s$ is defined as follows:

$$
\left.\begin{array}{rl}
\cos \theta_{2}<\cos \theta \leq 1 ; & s=\frac{x}{\cos \theta} \\
-\cos \theta_{1}<\cos \theta \leq \cos \theta_{2} ; & s=\frac{R}{\sin \theta} \\
-1 \leq \cos \theta \leq-\cos \theta_{1} ; & s=\frac{x-L}{\cos \theta}
\end{array}\right\}
$$

( $\cos \theta_{1}$ and $\cos \theta_{2}$ have the same meaning as above). Let $\phi\left(E, E^{\prime}\right) d E^{\prime}$ denote the probability, per unit path length, for an electron or positron of energy $E$ to produce a photon in the energy interval $\left(E^{\prime}\right.$, $\left.E^{\prime}+d E^{\prime}\right),{ }^{19}$ and let $d E / d s$ be the average energy loss per unit path length of an electron or positron due to ionizing collisions. ${ }^{20}$ The probability of an escape of a bremsstrahlung quantum produced by an electron or positron of energy $E$ in the course of slowing down is

$$
\begin{aligned}
P_{2}(E)= & \frac{1}{R(E)} \int_{\hat{E}}^{E} d E^{\prime} \\
& \int_{\hat{E}}^{E^{\prime}} d E^{\prime \prime}\left[\phi\left(E^{\prime}, E^{\prime \prime}\right) /-\frac{d E}{d s}\left(E^{\prime}\right)\right] P_{1}\left(E^{\prime \prime}\right),
\end{aligned}
$$

where $R(E)$ is the electron or positron range, and $\hat{E}=50 \mathrm{kev}$ is the cutoff.

Let $\psi(k) d k$ be the probability that one of the members of the pair has acquired initially a fraction between $k$ and $k+d k$ of the total available kinetic energy. ${ }^{20}$ The probability of the escape of at least one photon in the form of bremsstahlung emitted by a pair of total energy $E$ while slowing down is

$$
\begin{aligned}
P_{3}(E)=\int_{0}^{1} d k \psi(k)\left[P_{2}(k E)+\right. & P_{2}(E-k E) \\
& \left.-P_{2}(k E) P_{2}(E-k E)\right] .
\end{aligned}
$$

${ }^{19}$ H. Bethe and W. Heitler, Proc. Roy. Soc. [A] 146, 83 (1934)。 ${ }^{20} \mathrm{H}$. Bethe, Handbuch der Physik, 24, 519 (1933). 
The above formula assumes that each member of the pair can emit at most one photon. with energy greater than $50 \mathrm{kev}$ in the course of slowing down. We believe that the effects of plural photon production can be safely neglected in the energy range of interest here.

Combined Effect. The probability that the absorption of a primary photon of energy $E$ in a first interaction will be spurious due to the combined effects of bremsstrahlung and annihilation radiation is

$$
\begin{aligned}
P_{4}(E)= & \frac{\mu_{\mathrm{PH}}}{\mu_{\mathrm{PH}}+\mu_{\mathrm{P}}} P_{2}(E) \\
& +\frac{\mu_{\mathrm{P}}}{\mu_{\mathrm{PH}}+\mu_{\mathrm{P}}}\left[P_{3}\left(E-2 m c^{2}\right)+P_{0}-P_{3}\left(E-2 m c^{2}\right) P_{0}\right] .
\end{aligned}
$$

In evaluating this formula we found that the neglect of bremsstrahlung (i. e., setting $P_{2}$ and $P_{3}=0$ ) would lower $P_{4}$ by only a few percent (a maximum of $\sim 11 \%$ for crystal No. 9 at $4.45 \mathrm{Mev}$ ). The probability, per incident photon, of a spurious absorption in the first interaction is

$$
P_{6}(E)=[Y(E) / \mu(E)]\left[\mu_{\mathrm{PH}}(E)+\mu_{\mathrm{P}}(E)\right] P_{4}(E) .
$$

Next we must determine a corresponding probability of spurious absorption in a second interaction. One contribution to this probability is similar in origin to (21), and can be written:

$$
\begin{aligned}
P_{6}(E)= & {[Y(E) / \mu(E)] \int_{\hat{E}}^{E} d E^{\prime} \frac{\mu_{\mathrm{PH}}\left(E^{\prime}\right)+\mu_{\mathrm{P}}\left(E^{\prime}\right)}{\mu\left(E^{\prime}\right)} } \\
& k\left(E, E^{\prime}\right) Q\left(E^{\prime}\right) P_{4}^{*}\left(E^{\prime}\right),
\end{aligned}
$$

where $k\left(E, E^{\prime}\right)$ is the Klein-Nishina differential coefficient for Compton scattering with energy change from $E$ to $E^{\prime} ; Q$ is the probability that the scattered incident photon will not escape from the crystal without further interaction ${ }^{21}$; and $P_{4}^{*}(E)$ is the probability that a given absorption event is spurious, and is analogous to $P_{4}(E)$, but should take into account the fact that the source of secondary radiation is no longer concentrated on the crystal axis. We have ignored this circumstance, because the evaluation of an exact expression for $P_{4}^{*}(E)$ would be exceedingly complicated, and have set $P_{4}^{*}(E)=P_{4}(E)$. It is hoped that this will introduce only a minor error, for the following reasons: For small crystals, such a geometrical error might be serious, but a second interaction is quite rare; for large crystals, in which multiple interactions are more common, the geometrical

${ }^{21}$ An expression for $Q$ has been given by Maeder et al. (footnote 3, eq 44, 45, 46;) their $H_{0}$ is our $Y Q$. error is much less significant. In any case, the contribution of $P_{6}$ to the change in the photofraction will be small, so that a rough-and-ready treatment is in order.

There is also the possibility that an absorption taking place in a second interaction is spurious because it was preceded by the escape of a bremsstrahlung quantum from a Compton recoil electron. The probability for this to happen is

$$
\begin{aligned}
P_{7}(E)= & {[Y(E) / \mu(E)] \int_{\hat{E}}^{E} d E^{\prime} \frac{\mu_{\mathrm{PH}}\left(E^{\prime}\right)+\mu_{\mathrm{P}}\left(E^{\prime}\right)}{\mu\left(E^{\prime}\right)} } \\
& k\left(E_{1} E^{\prime}\right) Q\left(E^{\prime}\right) P_{1}\left(E-E^{\prime}\right) .
\end{aligned}
$$

Thus a spurious interaction will occur with probability

$$
P_{8}(E)=P_{6}(E)+P_{7}(E)-P_{6}(E) P_{7}(E)
$$

per incident photon in a second interaction; and with probability

$$
P_{9}(E)=P_{5}(E)+P_{8}(E)-P_{5}(E) P_{8}(E)
$$

in a first or second interaction. At the highest energy and for the largest crystal considered, the value of $P_{8}$ was only 15 percent of $P_{5}$, and generally it was much smaller. Subsequent interactions will lower the photon energy further and make spurious absorptions even more unlikely. Hence we felt justified in ending our calculations with the effects of the second interaction. The change in the photofraction is thus

$$
\Delta p=-P_{9}(E) Y(E) .
$$

\subsection{Effect on the Shape of the Response Function}

In considering the detailed shape of the response function we have calculated only the effects of annihilation radiation from a first interaction. Other escaping secondary radiation was taken into account only insofar as it shifted pulses from the photopeak into tail of the response function (i. e., by an appropriate renormalization of $\left.K\left(E, E^{\prime}\right)\right)$.

None, or one, or both of the annihilation quanta resulting from a pair production can escape from the crystal without further interaction. The probabilities for these contingencies can be obtained by replacing the integrand in the inner integral in (15) by the expressions
(a) (no escape) $:\left(i-e^{-s_{1} \mu^{\prime}}\right)\left(1-e^{-s_{2} \mu^{\prime}}\right)$
(b) (one escape) $: e^{-s_{1} \mu^{\prime}}\left(1-e^{-s_{2} \mu^{\prime}}\right)+e^{-s_{2} \mu^{\prime}}$ $\left(1-e^{-s_{1} \mu^{\prime}}\right)$
(c) (two escapes): $e^{-s_{1} \mu^{\prime}} e^{-s_{2} \mu^{\prime}}$. 
In case (a) the energy $E^{\prime}$ left in the crystal is the sum of three terms; two of them are each distributed as

$$
L\left(m c^{2}\right) / Y(E)=K\left(m c^{2} E^{\prime}\right)+p\left(m c^{2}\right) \delta\left(E^{\prime}-m c^{2}\right)
$$

the third contribution is a constant amount of energy $E-2 \mathrm{mc}^{2}$.

In case (b), $E^{\prime}$ is the sum of two terms, one distributed according to (29), the other a constant equal to $E-2 \mathrm{mc}^{2}$. In case (c) $E^{\prime}=E-2 \mathrm{mc}^{2}$. The correction of the response function was performed by shifting the appropriate number of pulses from the photopeak to the "tail" of the pulse height spectrum, distributing them according to the distribution functions described above weighted according to (28).

Washington, November 4, 1955. 